Scaramuzzi, F., Gas loading of deuterium in palladium at low temperature. J. Alloys and Compounds, 2004. 385: p. 19.

\title{
Gas loading of deuterium in palladium at low temperature
}

\author{
F. Scaramuzzi \\ INFN-LNF, Via E. Fermi 40, 00044 Frascati (Rome), Italy
}

Received 12 February 2004; received in revised form 20 April 2004; accepted 20 April 2004

\begin{abstract}
The experimental technique presented in this article is aimed at measuring the absorption of hydrogen or deuterium gas in a thin palladium sample while the system is at low temperature. A result for deuterium is described, consisting in the measurement of the equilibrium loading ratio $X$ (called also D/Pd ratio, atomic), as a function of pressure, on a palladium film $3.6 \mu \mathrm{m}$-thick at $150 \mathrm{~K}$. Values of $X$ up to 1 have been measured at pressures lower than 1 bar. The electric resistance of the palladium sample also has been measured as a function of temperature and of $X$, and the results are reported.
\end{abstract}

Keywords: Hydrogen storage materials; Metals; Thin films; Gas-solid reactions; Low temperatures

\section{Introduction}

Let us first define a quantity which we will need frequently in the following: the loading ratio $X$, called also $\mathrm{H} / \mathrm{Pd}(\mathrm{D} / \mathrm{Pd})$ ratio, which is the atomic ratio between $\mathrm{H}(\mathrm{D})$ and $\mathrm{Pd}$ in the compound. Loading hydrogen or deuterium in palladium to high values of $X$ has been pursued for decades, initially in order to investigate the particular behavior of this system as far as superconductivity is concerned. It is well known that palladium is not superconducting, while its hydride (deuteride) shows a superconducting transition if $X$ exceeds a critical value of about 0.8 , and the critical temperature increases as $X$ increases [1]. More recently the need for high loading ratios $(\geq 0.9)$, in particular for deuterium, has been claimed as a necessary condition in order to investigate some of the phenomena known under the name of cold fusion [2].

Obtaining loading ratios higher than 0.7 is not easy. The value of $X$ at equilibrium is a function of pressure and temperature. Fig. 1a, taken from ref. [3], shows a set of isotherms for palladium deuteride, in which the value of $X$ at equilibrium is plotted as a function of pressure $P$. It is evident that $X$ increases with increasing pressure and decreasing temperature. In order to attain high values of $X$, different methods have been used, such as electrolysis (also at low temperatures), gas loading at high pressures (up to tens of kbar) and ion implantation; they are thoroughly reported in ref. [1].

Fig. $1 \mathrm{~b}$ has been obtained by cutting the graphs in Fig. 1a with an isobar at 652 torr, and shows the behavior of $X$ as a function of temperature. The result is encouraging, in view of attaining high loading ratios; it has been the starting point for this experiment. A preliminary note, mainly dedicated to testing the cryogenics of this experiment, has been published earlier [4]. 
When working at low temperatures, the diffusion coefficient of hydrogen (deuterium) in palladium becomes much smaller, and reaching equilibrium can require long times. This problem has been faced by one of the features of the method presented here. With it, it has been possible to extend the measurements as long as necessary, until thermodynamic equilibrium was reached, even though many days were necessary for each experimental point.

Fig. 2 describes schematically the method used: a volume $V_{0}$ of about $20 \mathrm{~cm}^{3}$, is connected through a capillary to the cell $\mathrm{C}$ (volume of about $3 \mathrm{~cm}^{3}$ ), in which the sample is located. The gas in $V_{0}$ is kept at constant (almost) room temperature, while the cell can be cooled to temperatures down to $30 \mathrm{~K}$ (in the present configuration); its temperature can be held constant for long times (up to weeks, months). The experiment consists of putting a known amount of deuterium gas in the system, and then deducing from the measurement of the pressure $P$ the amount of absorbed deuterium, and thus the loading ratio $X$.
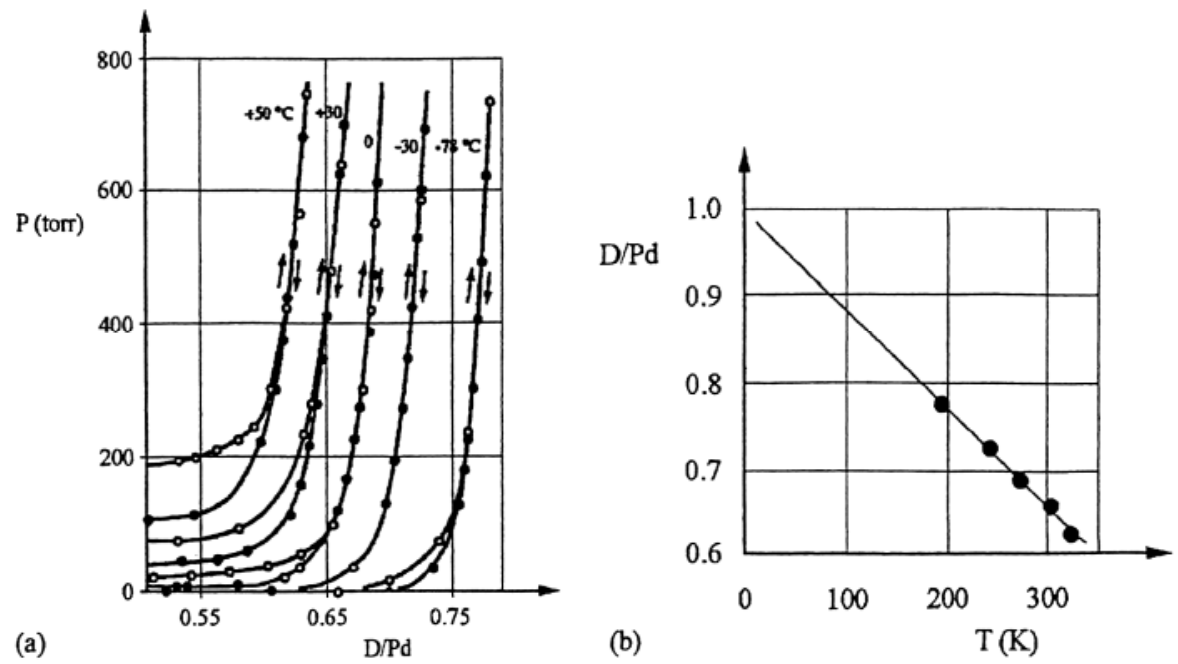

Figure 1. The equilibrium $\mathrm{D} / \mathrm{Pd}$ ratio for deuterium in palladium as a function of pressure and temperature: (a) is taken from ref. [3] and (b) is obtained by cutting (a) with an isobar at 652 torr.

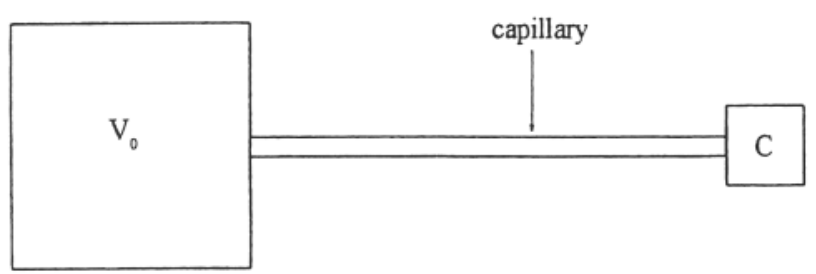

Figure 2. Scheme of the method used for the measurement of the D/Pd ratio. 


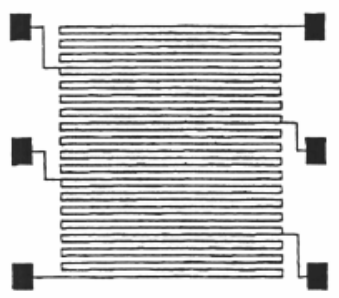

Figure 3. The "boustrophedonic" shape of the palladium serpentine. Note the six pads for the electrical connections.

\section{Description of the apparatus}

\subsection{The sample}

The sample is a thin film of palladium, deposited by IBAD (Ion Beam Assisted Deposition) ${ }^{1}$ on a $1 \mathrm{~mm}$-thick glass plate of $25 \mathrm{~mm} \times 35 \mathrm{~mm}$, on which a very thin layer of chromium had been previously deposited (rf-sputtering, $400 \AA$ ). Patterning of the sample is carried out using a previously fabricated mask for optical lithography; standard chemical etching techniques are used to obtain the pattern, which has the shape of a "boustrophedonic" serpentine (see Fig. 3). This geometry permits the measurement of the electrical resistance of palladium as a function of temperature and of loading ratio, for each of the five sections in which the serpentine is divided. Here are the data of the serpentine: length $2.5 \mathrm{~m}$, width $100 \mu \mathrm{m}$, thickness $3.6 \mu \mathrm{m}$, mass $12.1 \mathrm{mg}$, electric resistance of the unloaded sample at room temperature about $1000 \Omega$.

\subsection{The cryostat}

A closed-circuit gas refrigerator, of the Cryodyne type ${ }^{2}$ has been used. It produces refrigeration at two stages, with the following typical (indicative) values: the first stage can absorb a thermal power of $50 \mathrm{~W}$ at $50 \mathrm{~K}$, the second stage a power of $5 \mathrm{~W}$ at $20 \mathrm{~K}$. With no thermal charge applied, the second stage can reach the temperature of $10 \mathrm{~K}$. It is a rugged automated device, which can work up to 6 months without servicing.

Fig. 4 shows the scheme of the cryostat built around the Cryodyne refrigerator. An outer container $\mathrm{S}_{0}$ allows the vacuum insulation necessary in order to reach low temperatures. A small activated charcoal cryogenic pump is thermally attached to the second stage of the Cryodyne. Once a preliminary vacuum has been obtained by a rotary pump, the connection with the pump is closed and the vacuum is insured by the charcoal adsorption; a pressure of $10^{-6}$ mbar can be maintained for months. Inside $S_{0}$ a first shield $S_{1}$ (aluminum) is thermally attached to the first stage of the Cryodyne: the scope is to drastically reduce the heat inlet from the outer can $\mathrm{S}_{0}$, which is mainly by radiation. A second shield $S_{2}$ (copper) is connected with the second stage of the Cryodyne through a calibrated thermal link $\mathrm{R}_{1}$ (a copper wire of appropriate size). With the help of a thermometer, of a heater, and of a commercial thermoregulator ${ }^{3}$ it is possible to keep this shield at constant temperature at any value between 300 and about $20 \mathrm{~K}$. The cell, made out

\footnotetext{
${ }^{1}$ The sample was fabricated by Sandro Barbanera, whom the author takes the occasion for thanking, at the "Institute of Photonics and Nanotechnologies" of the Consiglio Nazionale delle Ricerche (CNR) at Rome.

${ }^{2}$ CTI-Cryogenics, Model 1020C Cryodyne Cryocooler.

${ }^{3}$ Lake Shore Cryotronics Inc., Model 340 Temperature Controller.
} 
of copper, is positioned inside $\mathrm{S}_{2}$ and is connected to it by another thermal link, $\mathrm{R}_{2}$. Also on the cell there are a thermometer, a heater and a thermoregulator.

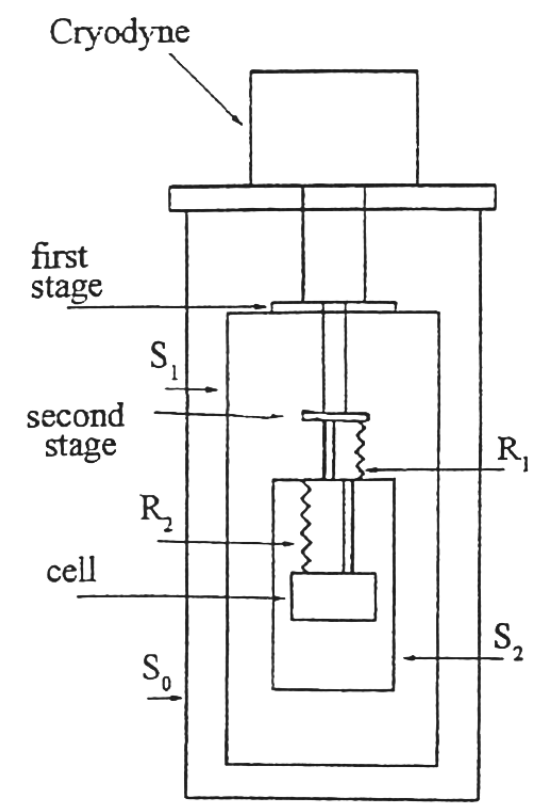

Figure 4. The scheme of the cryostat.

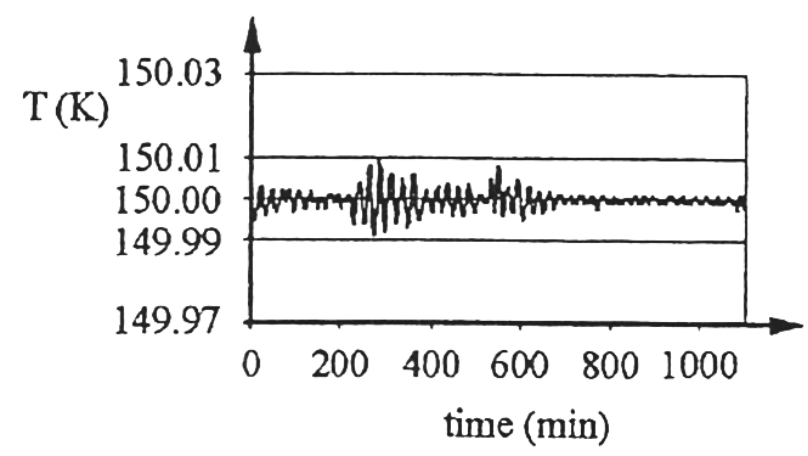

Figure 5. The cell thermoregulation for a period of about 1 day.

The idea is to keep the cell at a temperature slightly higher than that of $\mathrm{S}_{2}$. The presence of the thermoregulated $S_{2}$ shield insures a more efficient thermoregulation of the cell. A graph of the cell temperature as a function of time for a time duration of roughly 1 day is shown in Fig. 5. This system permits to keep the cell at any temperature between room temperature and about $30 \mathrm{~K}$ for long periods. A simple modification (the addition of a third shield) would permit to attain temperatures close to $10 \mathrm{~K}$, and the use of a more modern refrigerator would permit temperatures close to $4 \mathrm{~K}$.

The cell is made of two massive copper flanges, joined with the help of an indium vacuum gasket. The glass plate on which palladium is deposited is placed in its center; this allows dismantling the cell without touching the rest of the gas circuit. The six electrical connections for the resistance measurements are realized through ceramic-to-metal feedthroughs with the help of indium gaskets placed between the feedthrough and the palladium pad (see Fig. 3). 


\subsection{The gas circuit and the measuring protocol}

Fig. 6 shows in detail the gas circuit, and helps understanding its logic. The thermoregulated box contains the two valves (pneumatically operated) $v_{i}$ and $v_{e}$ and the head of the pressure meter. ${ }^{4}$ The volume $\mathrm{V}_{0}$ between the two valves has been measured. The capillary starts at $\mathrm{v}_{\mathrm{i}}$, enters the Cryodyne vacuum tank $\left(\mathrm{S}_{0}\right)$ and leads to the cell.

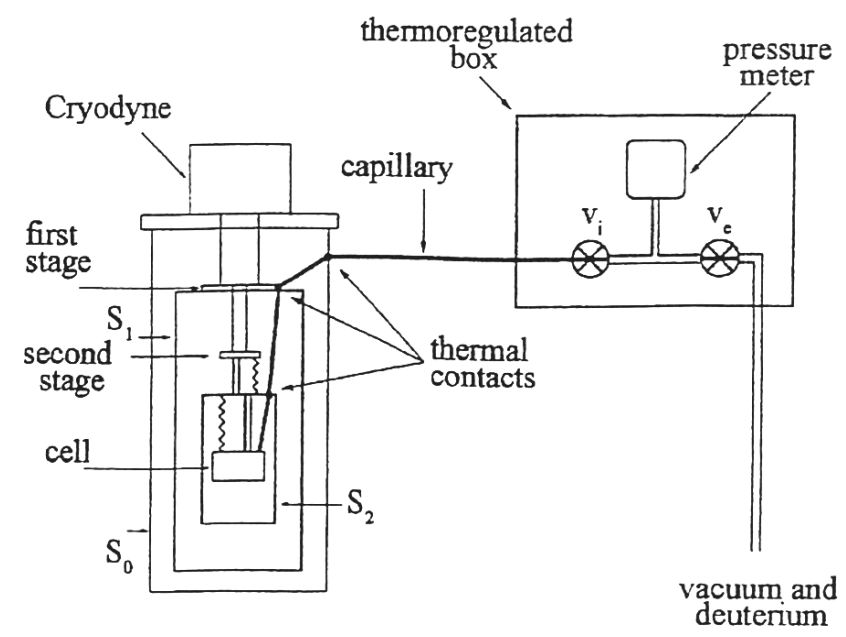

Figure 6. The gas circuit.

The volume of the capillary's two sections (from $v_{i}$ to $S_{0}$ to the cell) was measured during the set-up. The volume of the cell was measured as well, and this measurement was repeated every time the cell was disassembled.

The experiment described hereafter consisted in the measurement of the equilibrium $\mathrm{D} / \mathrm{Pd}$ ratio with the sample at $150 \mathrm{~K}$, for three different values of the pressure. The procedure was the following. Once the system is evacuated, valve $v_{i}$ is closed, and through valve $v_{e}$ deuterium gas is admitted to the system at the wanted pressure; then, $v_{e}$ is closed. At this point, all the gas admitted to the system is contained in $V_{0}$. Since volume, pressure and temperature are known, it is possible to calculate the amount of gas, say the number of moles $n$, contained in the system. Once the cell is stabilized at the wanted temperature (in this case at $150 \mathrm{~K}$ ), $\mathrm{v}_{\mathrm{i}}$ is opened. The gas will expand reaching the cell and will be partially absorbed by the palladium sample. It is possible to monitor the absorption by the change of pressure. Once the pressure has reached a value constant in time, we assume that the absorption is complete and that the system is in equilibrium. At this point $\mathrm{v}_{\mathrm{i}}$ is closed. Taking note of the amount of gas present in $V_{0}$, which can be deduced from the residual pressure, more gas is introduced through $\mathrm{v}_{\mathrm{e}}$ up to the second value chosen for the pressure. It is now possible to calculate the amount of gas added to the system. The sum of this quantity and that introduced for the first step gives the total amount of gas now present in the system. Then the same procedure described before is repeated, reaching a second value of the equilibrium pressure. In this experiment three points have been taken, corresponding to three values of $n$, and thus of $P$. These values of $P$ will permit the calculation of the $\mathrm{D} / \mathrm{Pd}$ ratio, as explained in the following.

\footnotetext{
${ }^{4}$ MKS Instruments Inc., measuring head MKS Baratron, Model 622A, 1000 torr full scale.
} 


\section{Description of the measuring method}

\subsection{Thermodynamics}

The principle of the measurement presented here is simple, but its realization requires a certain attention. We have stated that the number of moles of gas present in the system is known. Once $\mathrm{v}_{\mathrm{i}}$ is open, if there is no absorption, the gas will distribute itself in the circuit so as to satisfy its equation of state. If we know the distribution of temperature in the various volumes constituting the circuit, we can write this equation, and thus we are able to calculate the value that pressure $P$ will attain at equilibrium. If there is absorption, the pressure will reach a lower value. The difference between these two pressures, the one without absorption (calculated) and the one with absorption (measured), will permit the calculation of the amount of gas that has been absorbed. This, in turn, provided that we know the mass of palladium, will allow calculating the loading ratio $X$.

Let us analyze the geometry of the gas circuit (see Fig. 6) from the point of view of the temperature distribution. The content of the thermoregulated box, i.e., volume $V_{0}$ plus the section of the capillary running inside the box, are at a known constant temperature $T_{0}$. The section of capillary running from the box to $\mathrm{S}_{0}$ is exposed to room temperature; this temperature $\left(T_{1}\right)$ is (slowly) varying, and is measured and monitored. The section of capillary running from $\mathrm{S}_{0}$ to the cell is exposed to various temperatures. Its volume is about $0.4 \mathrm{~cm}^{3}$ over a total volume of the circuit of about $23 \mathrm{~cm}^{3}$. It is thus reasonable to use the following approximate solution. We consider two points along the capillary's path, of which we measure and monitor the temperature; these are the first stage of the Cryodyne and the shield $S_{2}$. We create thermal contacts between these points and the capillary (see Fig. 6). In so doing we have divided this part of the capillary in three subsections. We make the hypothesis that we can consider these subsections as three volumes at constant (average) temperature, the temperature being the arithmetic average between the extreme temperatures of each subsection. With this hypothesis, and remembering that the cell is at constant known temperature, the whole system is divided in six sections, two at constant temperature (box and cell), one at slowly varying but known temperature, and three small ones for which we assume an average temperature; for each section we know volume and temperature. Let us now write the state equation for each of these sections; in a first approximation we will use the ideal gas equation, and we will justify it later (see Appendix A). We will attribute to the six volumes and to the corresponding temperatures the subscripts from 0 to 5. Describing an equilibrium condition, we can write the following equations:

$$
\sum_{i} n_{i}=n \quad n_{i}=\frac{P V_{i}}{R T_{i}} \quad(i=0, \ldots, 5)
$$

It is a system of seven equations in the seven unknowns $n_{0}, \ldots, n_{5}, P$. The equation on the left is the expression of a mass conservation principle: the sum of the masses contained in each volume, expressed as number of moles, must be equal to the total mass present in the system, $n$, which we know. What we are interested in is the value of $P$. If we substitute the six equations on the right in the one on the left, we obtain:

$$
P=\frac{n R}{\sum_{i}\left(V_{i} / T_{i}\right)} \quad(i=0, \ldots, 5)
$$


A test has been performed in order to check the validity of the approximation here described, and, in general, the validity of the method. A "blank" experiment has been performed, in which there was no palladium. It consisted of introducing deuterium gas in the system, and measuring its amount (which was $n=6.86 \times 10^{-4} \mathrm{~mol}$ ). Then the temperature of the cell was held constant at various values, as long as necessary, so that thermodynamic equilibrium could be reached. This was done at the temperatures of 300, 250, 200, 150, 102 and $45 \mathrm{~K}$ while cooling the cell, and was repeated at the same values while returning to room temperature. The whole experiment lasted 1 week. The results of this measurement are summarized in Table 1.

The first two rows show the values of pressure measured in the two series of measurements, going down $\left(P_{\mathrm{m} \downarrow}\right)$ and up $\left(P_{\mathrm{m} \uparrow}\right)$ in temperature. In the third row the average between them is calculated $\left(P_{\mathrm{m}}\right)$. In the fourth row is reported the calculated value of the pressure, $P_{\mathrm{c}}$. (In the calculation the effect of the thermal expansion of the system, mainly of the cell, was taken into account.) In the last two rows the measured and the calculated values are compared. We note that:

1. The difference between the values measured in the two series is less than $0.3 \%$.

2. The difference between measured and calculated values is less than $1 \%$.

This result is quite satisfactory. The first statement shows that the measurements are well reproducible. The second has to be looked at in the light of the intrinsic errors connected with such a measurement. In fact, in order to calculate $P$, absolute values of $T$ and $V$ must be used. These, as well as the measurement of $P$, are obtained by instruments characterized by an absolute error. The sum of the errors referring to the three quoted quantities, as indicated by the constructors, amounts to about $1 \%$. Thus, the result is well compatible with the characteristics of the instruments. We can conclude that the method proposed for the calculation of the pressure is thus validated.

Table 1

Check of the proposed calculation method: comparison between measured $\left(P_{\mathrm{m}}\right)$ and calculated $\left(P_{\mathrm{c}}\right)$ values of the pressure $P$; pressures are in mbar

\begin{tabular}{llllllc}
\hline & $T(\mathrm{~K})$ & & & & & \\
\cline { 2 - 7 } & 300 & 250 & 200 & 150 & 102 & 45 \\
\hline$P_{\mathrm{m} \downarrow}$ & 754.6 & 731.0 & 699.2 & 652.3 & 581.6 & 411.0 \\
$P_{\mathrm{m} \uparrow}$ & 752.5 & 729.0 & 698.5 & 652.0 & 581.7 & \\
$P_{\mathrm{m}}$ & 753.55 & 730.0 & 698.85 & 652.15 & 581.65 & 411.0 \\
$P_{\mathrm{c}}$ & 758.0 & 735.3 & 704.0 & 656.5 & 583.6 & 411.1 \\
$\Delta P=P_{\mathrm{c}}-P_{\mathrm{m}}$ & 4.45 & 5.3 & 5.15 & 4.35 & 1.95 & 0.1 \\
$\Delta P / P(\%)$ & 0.59 & 0.73 & 0.74 & 0.67 & 0.34 & 0.02 \\
\hline
\end{tabular}




\subsection{Data acquisition}

A Lab View system has been used, in order to permit recording the following quantities as a function of time:

- The temperature of the thermoregulated box, $T_{0}$.

- The ambient temperature, $T_{1}$.

- The temperature of the first stage of the Cryodyne, $T_{\mathrm{c} 1}$.

- The temperature of the $S_{2}$ shield, $T_{\mathrm{S} 2}$.

- The cell temperature, $T_{\mathrm{c}}=T_{5}$.

- The pressure in the system, $P$.

- The resistance of the five sections of the palladium serpentine: $R_{1}, R_{2}, R_{3}, R_{4}, R_{5}$ (the last section, R5, was broken a little time after being exposed to deuterium, and was shortcircuited).

The normal acquisition frequency was 1 reading/min, but occasionally also other values were used, from 1 reading/s to 1 reading/5 min.

\section{The experiment}

\subsection{Measurement of the loading ratio}

The main feature of the experiment consisted in the measurement of the deuterium absorption, i.e., the atomic D/Pd ratio, established in equilibrium in the palladium sample, while it was kept at the temperature of $150 \mathrm{~K}$. The cell was held at this temperature without interruption for the whole duration of the experiment, which was about 1 month, with the temperature constant within $\pm 0.01 \mathrm{~K}$. The amount of deuterium absorbed, expressed as a change $\Delta n$ of the number of moles of gas, $n$, present in the system, was calculated starting from the difference $\Delta P$ between the calculated pressure and its measured value. In order to do so, formula (1) of Section 3.1 was used. The $\mathrm{D} / \mathrm{Pd}$ ratio was measured in equilibrium for three values of the pressure $P$, corresponding to three values of $n$, the number of moles present in the system. Hereafter these measurements are briefly described:

- First point: $n_{1}=1.34 \times 10^{-4}$ mol. Once the palladium sample was exposed to the gas, it was necessary to wait for 9 days in order to reach equilibrium, i.e., a constant value of the pressure; its final value was $P_{1}=89.9$ mbar. Fig. 7 shows the variation of the pressure in this time interval. The value of the loading ratio resulted to be $\mathrm{D} / \mathrm{Pd}=0.743$.

- Second point: $n_{2}=4.76 \times 10^{-4} \mathrm{~mol}$ (this is the total mass of gas present in the system). After admitting more gas into the cell, 8 more days were necessary in order to reach equilibrium. The final value of the pressure was $P=418.4 \mathrm{mbar}$. The value of the loading ratio resulted to be $\mathrm{D} / \mathrm{Pd}=0.885$.

- Third point: $n_{2}=9.43 \times 10^{-4} \mathrm{~mol}$. Also in this case it was necessary to wait for 8 more days in order to reach equilibrium. The final value of the pressure was $P=869.0 \mathrm{mbar}$. The value of the loading ratio resulted to be $\mathrm{D} / \mathrm{Pd}=1.023$.

These results are summarized in Fig. 8, in a graph of the D/Pd ratio as a function of the pressure $P$. The error bars will be explained below (see Appendix B). 


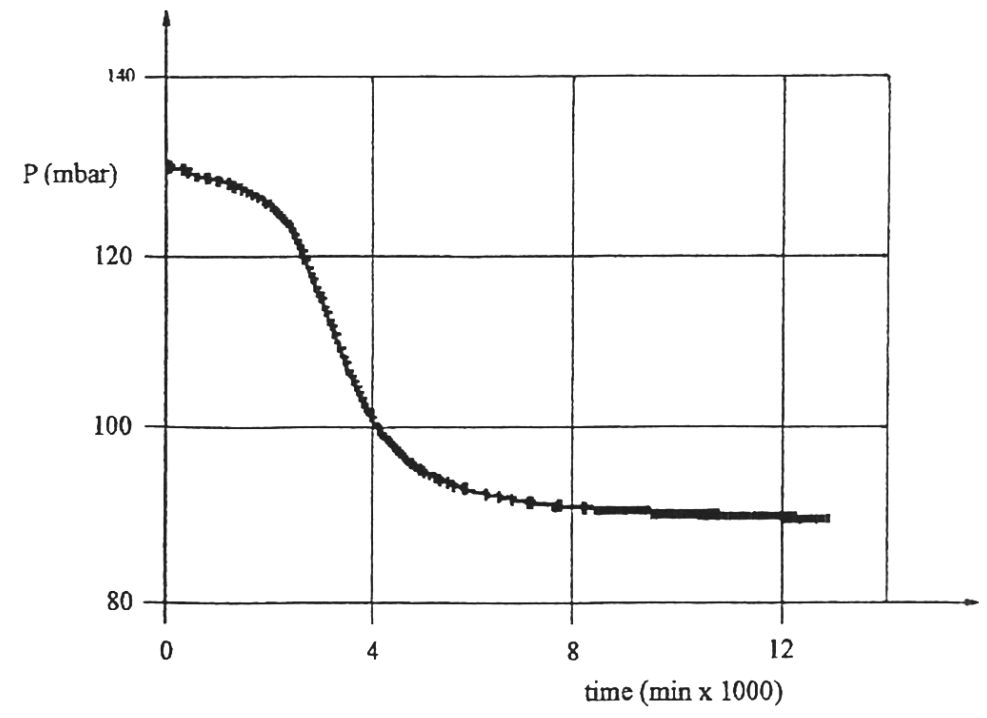

Figure 7. The pressure $P$ as a function of time in the 9 days relative to the first point.

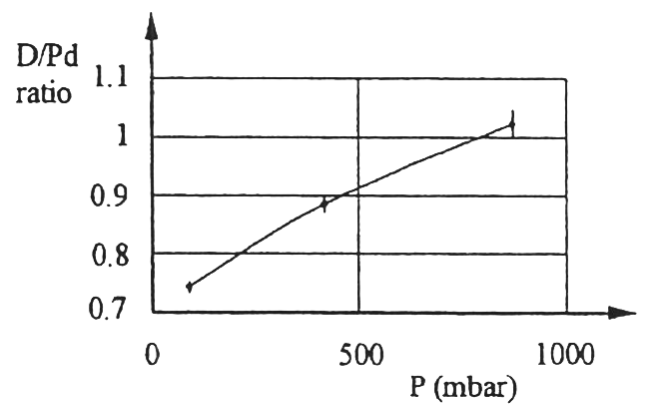

Figure 8. The result of this experiment in a graph showing the D/Pd ratio as a function of the pressure $P$ at $150 \mathrm{~K}$.

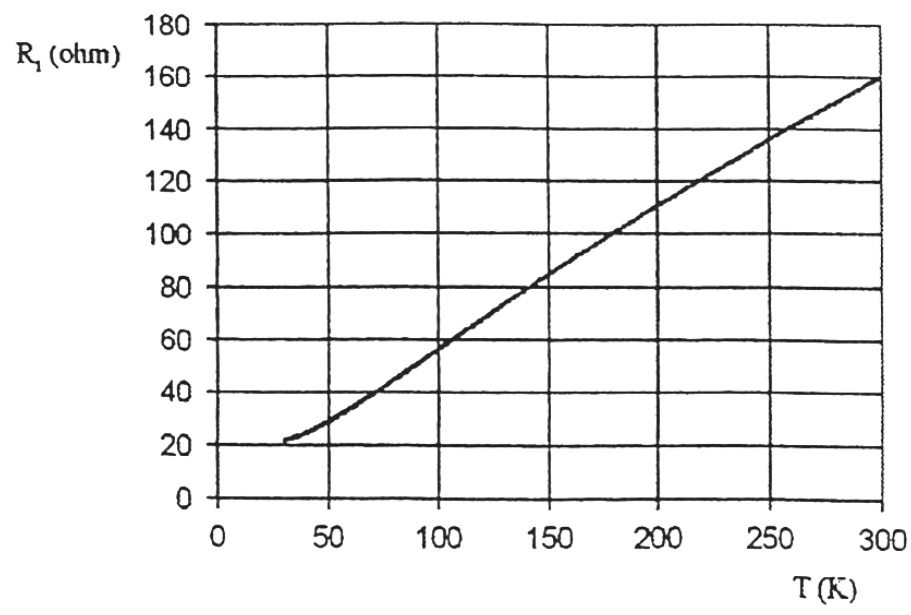

Figure 9. The electrical resistance of section $R_{1}$ as a function of temperature $T$, in the absence of absorbed deuterium. 


\subsection{Measurement of the electrical resistance}

Before admitting deuterium gas into the cell, a measurement of the electrical resistance of the sample as a function of temperature was performed in the interval 300-30 K. The measurement has been made in a dynamic mode, while the temperature was changing, though very slowly, in a first ramp down from 300 to $30 \mathrm{~K}$, and then in a ramp up from 30 to $150 \mathrm{~K}$. The whole measurement lasted $25 \mathrm{~h}$. The result for the first section $\mathrm{R}_{1}$ is shown in Fig. 9 (the other sections behave in a similar way). Note that in the same graph both series of data are reported, those in the ramp down and those in the ramp up, and it is not possible to perceive a difference between the two. This shows that the choice of a dynamical mode did not influence appreciably the results.

In Fig. 10a the resistance of the same section $\mathrm{R}_{1}$ is reported as a function of time during the first loading episode. It is not possible to correlate the value of the resistance with that of the $\mathrm{D} / \mathrm{Pd}$ ratio, but for the final equilibrium condition. It is however suggestive to note that towards the end of the episode the value of the resistance reaches a maximum, and then starts to drop; this is better shown in Fig. 10b, representing an enlarged section of Fig. 10a. It is well known [5] that at room temperature the resistance of a $\operatorname{PdD}_{X}$ sample reaches a maximum for $X \approx 0.75$, and this is the value that the loading ratio was approaching in this experiment towards the end of the first episode.
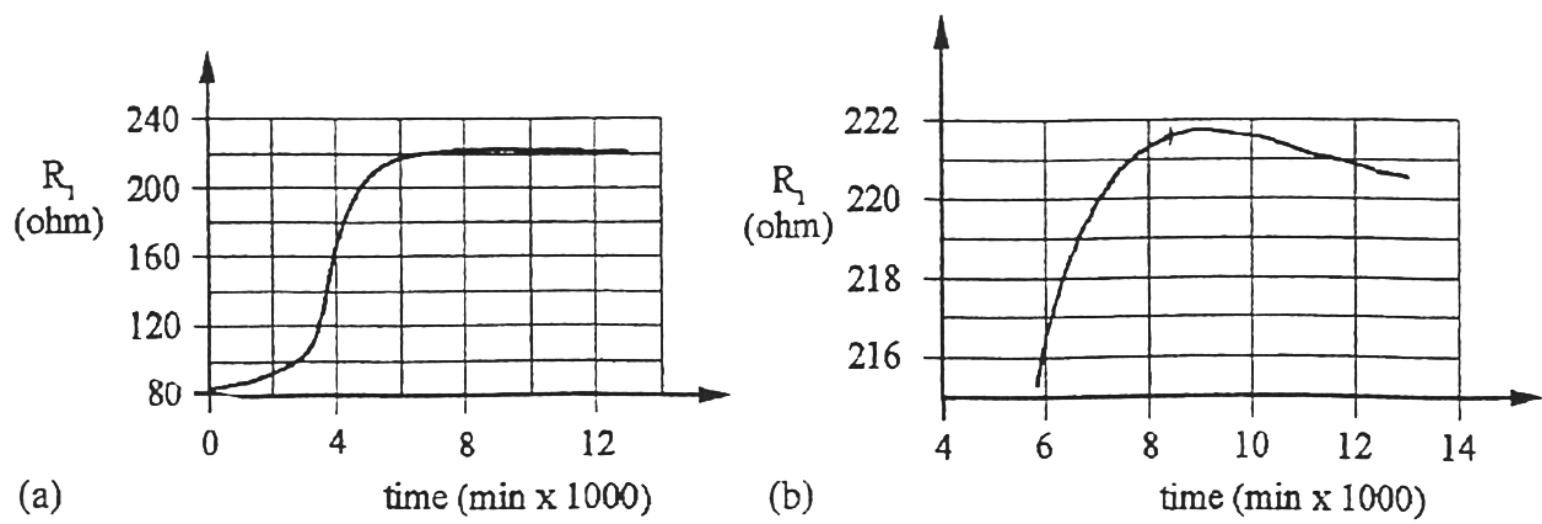

Figure 10. (a) The variation of the resistance R1, as a function of time, during the 9 days of the first episode of loading, (b) An enlarged section of the previous graph, showing clearly the maximum in the curve.

The ratio between the maximum value of the resistance, $R_{\max }$, and the value of the resistance in the absence of absorbed deuterium, $R_{0}$, is $R_{\max } / R_{0}=221.7 / 84.1=2.64$ : this value is, as expected, rather higher than the value measured at room temperature, which is about 2.

The values of the resistance of the five sections in which the sample is divided are summarized in Table 2.

The first two columns report the values of the resistance of the unloaded sample, i.e., the sample without deuterium, at 300 and at $150 \mathrm{~K}$. The following three columns report the value of the resistance of the loaded sample, at $150 \mathrm{~K}$, at equilibrium, for the three points described above. The last column gives the maximum value attained by the loaded sample (just before the end of the first point).

Fig. 11 shows a graph of the resistance $\mathrm{R}_{1}$ as a function of the $\mathrm{D} / \mathrm{Pd}$ ratio. The curve shown was constructed on the few experimental points at hand. 
Table 2

The electrical resistance (in ohms) of the sections in which the palladium sample is divided (see Fig. 3), as a function of temperature and D/Pd ratio: the first two columns on the left refer to the unloaded sample, at 300 and $150 \mathrm{~K}$; the following four columns refer to the loaded sample at $150 \mathrm{~K}$, with reference to the three points described above, plus the maximum value attained, $R_{\max }$

\begin{tabular}{|c|c|c|c|c|c|c|}
\hline \multicolumn{3}{|c|}{ Unloaded sample } & \multicolumn{4}{|c|}{ Loaded sample at $150 \mathrm{~K}$} \\
\hline & $300 \mathrm{~K}$ & $150 \mathrm{~K}$ & First point & Second point & Third point & $R_{\max }$ \\
\hline $\mathrm{R}_{1}$ & 159.7 & 84.1 & 220.5 & 217.2 & 214.3 & 221.73 \\
\hline $\mathrm{R}_{2}$ & 211.8 & 110.8 & 292.9 & 289.1 & 285.3 & 294.67 \\
\hline $\mathrm{R}_{3}$ & 207.2 & 108.0 & 286.4 & 282.9 & 279.2 & 289.0 \\
\hline $\mathrm{R}_{4}$ & 238.0 & 124.6 & 332.3 & 327.9 & 323.5 & 334.2 \\
\hline $\mathrm{R}_{5}$ & 199.1 & 107.7 & - & - & - & - \\
\hline
\end{tabular}

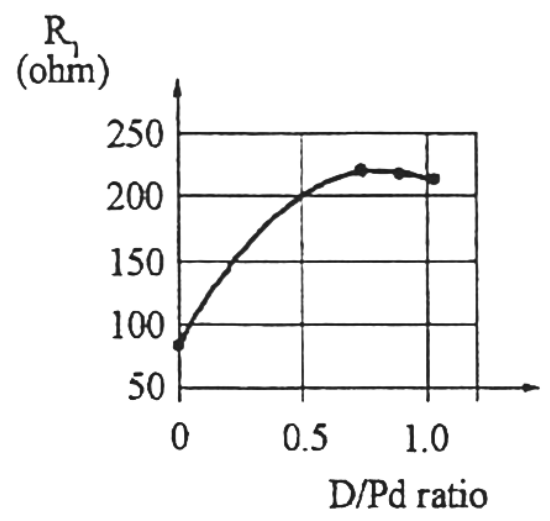

Figure 11. The resistance $R_{1}$ as a function of the D/Pd ratio.

\section{Future perspectives and conclusions}

The measurements reported in this article suggest a few considerations and indicate potential trends for the technique introduced here.

a. There are two main reasons for the choice of the temperature of $150 \mathrm{~K}$ for the experiment reported here. For one thing, it was possible to use the equation of state for ideal gases, without worrying about corrections, as shown in Appendix A. In addition, lower temperatures could present longer absorption times, due to the sharp decrease of the coefficient of diffusion. A guideline was the result presented in ref. [6], which shows that absorption is practically absent at temperatures lower than $150 \mathrm{~K}$, even though the data presented there are taken in different conditions, i.e., at high pressures and in a dynamical mode, while the temperature is changing at a rate of $2 \mathrm{~K} / \mathrm{min}$. The choice of $150 \mathrm{~K}$ was considered a good compromise for this measurement. Nevertheless, it would be interesting to investigate the behavior of such a system at lower temperatures.

b. One of the most interesting features of the method described in this paper is the possibility of performing measurements under constant conditions for quite long times. This has made it possible to assure that thermodynamic equilibrium had been attained by the system, and that the distribution of deuterium in palladium was rather uniform, an 
important condition in order to give a meaningful value of the $\mathrm{D} / \mathrm{Pd}$ ratio. A comparison with previous data can be performed by looking at Fig. 1b. The isobar from [3] shown in this graph is purposely taken at the pressure of 652 torr, corresponding to $869.0 \mathrm{mbar}$, the pressure of the third point presented in this paper. It can be noted that in the graph the value expected for $X$ at the temperature of $150 \mathrm{~K}$ is $X \approx 0.83$, much lower than the value $X \approx 1.02$ found in this experiment. It is reasonable to cast doubts on the uniformity of the deuterium distribution in the samples of ref. [3]; a higher concentration of deuterium in the outer layers of the sample would justify such an error. This is supported by the fact that the sample used in that measurement was $300 \mu \mathrm{m}$-thick, much more than the $3.6 \mu \mathrm{m}$ of the sample used in this experiment. This in turn suggests to check with this method the critical temperature $T_{\mathrm{c}}$ for the superconductive transition of $\mathrm{PdH}_{X}$ and $\mathrm{PdD}_{X}$; the use of a more modern refrigerator, able to reach the temperatures of liquid ${ }^{4} \mathrm{He}$, would permit performing this check in an easy way.

c. It is interesting that it has been possible to attain, at the temperature of $150 \mathrm{~K}$, the value 1 for the $\mathrm{D} / \mathrm{Pd}$ ratio at a pressure lower than 1 bar. It is reasonable to hope that it could be possible to obtain higher values at higher pressures: this would permit investigating the presence of a third phase, the $\gamma$-phase, of the palladium-deuterium (hydrogen) compound, which has been described in the literature [7].

d. The behavior of the electrical resistance as a function of the D/Pd ratio is at the same time comforting and slightly surprising. On the one hand, there is a confirmation of the value of $X$ corresponding to the maximum in the curve of Fig. 11. It is worthwhile to quote the paper by Geerken and Griessen [8], where it is stated that for all temperatures below 300 $\mathrm{K}$ the maximum takes place at $X \approx 0.75$, and graphs are shown with this feature at 300,20 and $4.2 \mathrm{~K}$. On the other hand, the further decrease of the resistance beyond the maximum is much smaller in this experiment than what is reported at room temperature.

e. The curve shown in Fig. 7, representing the change in pressure of the system in the presence of absorption, and in particular its final "exponential" section, could be useful to get information about the coefficient of diffusion of deuterium in palladium, as a function of temperature, of pressure and of $X$, provided that a reasonable model is taken into consideration.

\section{Acknowledgements}

The author is grateful to the Fusion Division of the ENEA Frascati Research Center for permitting the experiment described in this paper to be carried out in its laboratories. In particular, the author is deeply grateful to Luciano Mori, Fabrizio Marini, Luigi Verdini and Miriam Veschetti for the help provided in the construction of the apparatus and in the management of the experiment. The author expresses also his gratitude to Antonella De Ninno, Antonio Frattolillo and Paolo Tripodi for useful discussions.

\section{Appendix A. Non-ideality of the gas}

The equation of state of ideal gases does not take into account the interactions between atoms (or molecules), except collisions. A well known equation of state that tries to correct the ideal gas equation, by evaluating the effect of interactions, can be written as follows:

$P V=n\left(R T+B P+C P^{2}+\cdots\right)$. 
where $B, C$, and the following coefficients take the name of "virial". The second virial coefficient, $B$, is relevant at low temperatures; the third, $\mathrm{C}$, is relevant at high pressures. Thus, for this experiment, we can restrict ourselves to consider $B$.

The dependence of $B$ on temperature for deuterium is reported in Fig. 12 [9]. It is evident that $B$ is positive, quite small and almost constant between 400 and $200 \mathrm{~K}$, then it crosses the zero at about $120 \mathrm{~K}$, and becomes negative with a much higher slope at lower temperatures. This feature, with the hope of not needing to make corrections to the equation of state, contributed to the choice of $150 \mathrm{~K}$ for this first experiment.

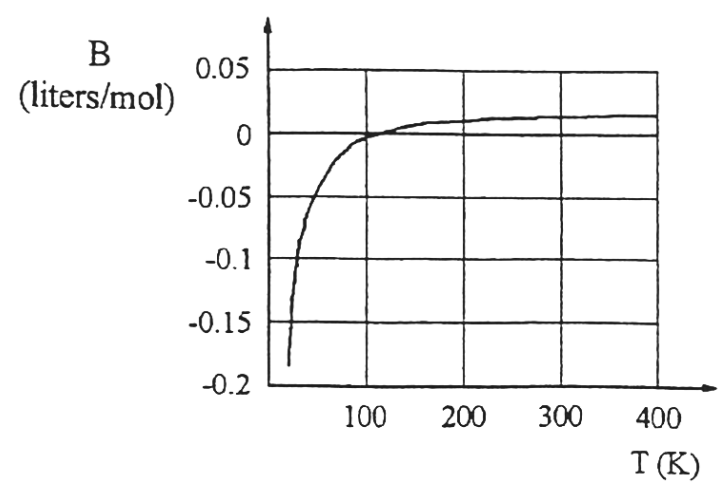

Figure 12. The second virial coefficient, $B$, for deuterium, as a function of temperature $T$.

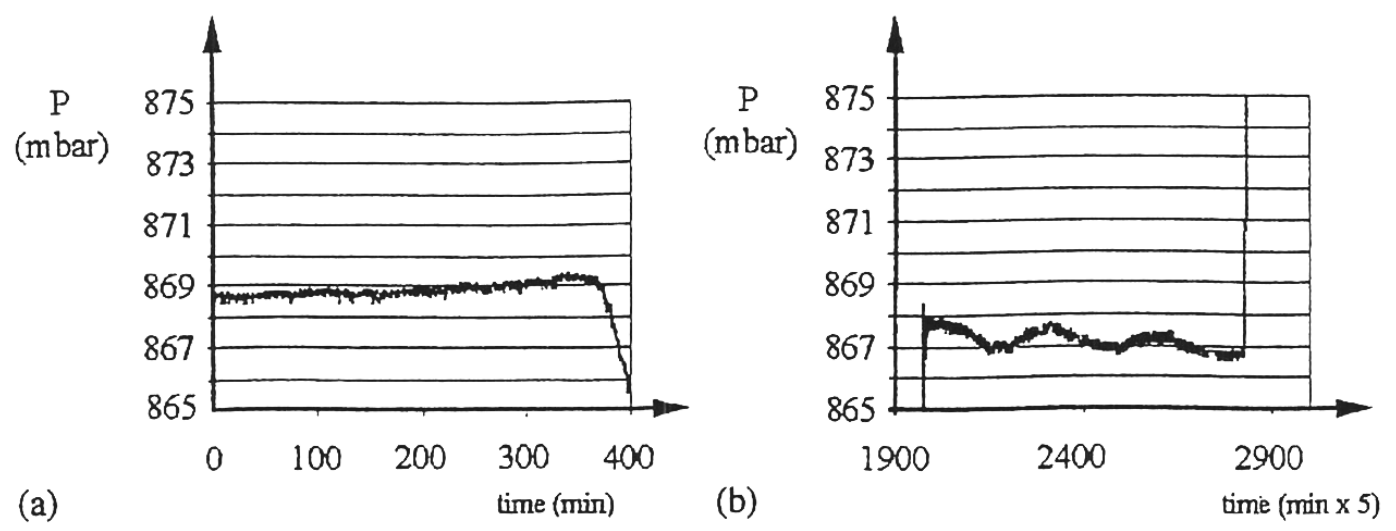

Figure 13. Comparison between values of the pressure in the system measured in the same experimental conditions at two different times. The first measurement (a) was taken at the end of the experiment. The second (b) was started 16 days later and extended for 3 more days. In the meanwhile the system had been the object of other tests at different temperatures (lower than $150 \mathrm{~K}$ ), and eventually brought again to $150 \mathrm{~K}$.

Table A.1

Comparison between the values of the pressure calculated following the equation of state of ideal gases, $P_{\text {ig }}$, and those obtained by taking into consideration the second virial coefficient, $P_{\text {vir }}$

\begin{tabular}{rrrrr}
\hline$T(\mathrm{~K})$ & $P_{\text {ig }}$ (bar) & $P_{\text {vir }}$ (bar) & $\Delta P($ bar $)$ & $\Delta P / P(\%)$ \\
\hline 300 & 0.8315 & 0.8319 & $4 \times 10^{-4}$ & 0.05 \\
\hline 30 & 0.3326 & 0.3298 & $2.8 \times 10^{-3}$ & 0.85 \\
\hline
\end{tabular}


However, in order to render the above considerations quantitative, a simple calculation was performed. Considering a situation very similar to the one used in the experiment (for volumes and geometry), and given a certain amount of gas inserted in the system, the equilibrium pressure was calculated both with the equation of state for ideal gases, $P_{\mathrm{ig}}$, and with the virial equation above written, stopped at the term in $B, P_{\text {vir }}$ for two temperatures, 300 and $30 \mathrm{~K}$. By comparing the two sets of results, reported in Table A.1, it can be noted that the correction is negligible at $300 \mathrm{~K}(0.05 \%)$, while at $30 \mathrm{~K}$ it starts to be relevant, with an error of $0.85 \%$.

It is reasonable to conclude that for this experiment the use of the equation of state of ideal gases is a correct choice.

\section{Appendix B. Sources of error}

In this section we will list a series of considerations intended to help the evaluation of the possible errors in the measurements presented here, in particular those relative to the $\mathrm{D} / \mathrm{Pd}$ ratio.

\section{B.1. Instrumental errors}

In Section 3.1 we have shown that the absolute errors due to the instruments used for the measurement of pressure, volume and temperature result in an error lower than $1 \%$ in the calculation of the pressure $P$. This error is transmitted in the determination of the $\mathrm{D} / \mathrm{Pd}$ ratio, becoming increasingly higher for each of the three loading episodes. In any case, it has been evaluated that it does not exceed $4 \%$ in the last loading.

\section{B.2. The sample}

The error in the determination of the mass of palladium is on the order of $1 \%$, and it has to be added to the instrumental errors.

\section{B.3. Consequences of a leak in the system}

This is a real risk. Imagine that the system has a leak, for example, towards the Cryodyne vacuum system. In time this would cause a decrease of the pressure in the system, thus altering the whole measurement. Besides the many satisfactory tests performed in order to check that the system was leak-tight, a further check is reported here. Fig. 13a shows the measurement of the pressure $P$ in the system as a function of time at the end of the experiment. In Fig. 13b the same measurement is taken after 16 days and extended for more than 3 days (after performing a certain number of checks on the system at lower temperatures, and eventually bringing the cell again up to $150 \mathrm{~K}$ ). The comparison between the two measurements shows a difference of less than 2 mbar on a value of 868 mbar, with an error of about $0.2 \%$ in 18 days. It is thus reasonable to exclude this feature as a possible cause of error.

The error bars shown in Fig. 8 have been evaluated in the light of what has been said in this section. 


\section{References}

[1] B. Stritzker, H. Wiihl, Superconductivity in Metal-Hydrogen Systems, a chapter in Hydrogen in Metals II (Application-Oriented Properties), Springer-Verlag, 1978, p. 243.

[2] M.C.H. McKubre, S. Crouch-Baker, A.M. Riley, S.I. Smedley, F.L. Tanzella, in: Proceedings of the 3rd International Conference on Cold Fusion, Nagoya, Japan, 21-25 October 1992, Universal Academic Press, Frontiers Science Series no. 4, 1993, p. 5.

[3] E. Wicke, G.H. Nemst, Ber. Bunsenges. Phys. Chem. 68 (1964) 224.

[4] E. Del Giudice, A. De Ninno, A. Frattolillo, G. Preparata, F. Scaramuzzi, P. Tripodi, in: Proceedings of the 8th International Conference on Cold Fusion, Lerici (SP), Italy, 21-26 May 2000, Editrice Compositori, Bologna, Conference Proceedings no. 70 of the Italian Physical Society, 2000, p. 211.

[5] G. Bambakidis, R.J. Smith, D.A. Otterson, Phys. Rev. 177 (1969) 1044.

[6] H. Hemmes, A. Driessen, R. Griessen, M. Gupta, Phys. Rev. B 39 (1989)4110.

[7] S.A. Semiletov, R.V. Baranova, Yu.P. Khodyrev, R.M. Imamov, Sov. Phys. Crystallogr. 25 (6) (1980) 665.

[8] B.M. Geerken, R. Griessen, J. Phys. F: Met Phys. 13 (1983) 963.

[9] P. Clark Souers, Hydrogen Properties for Fusion Energy, University of California Press, 1986, p. 55. The author takes the occasion for thanking G.T. (Terry) McConville, Physics Department University of Cincinnati, for pointing out this text and for useful discussions. 